\title{
AUTOMATED BEAM BASED ALIGNMENT OF THE ALS QUADRUPOLES*
}

\author{
G. Portmann, D. Robin, and L. Schachinger \\ Advanced Light Source \\ Accelerator and Fusion Research Division \\ Lawrence Berkeley Laboratory \\ University of California \\ Berkeley, CA 94720
}

April 1995

Paper presented at the 1995 Particle Accelerator Conference, Dallas, TX, May 1-5, 1995

*This work was supported by the Director, Office of Energy Research, Office of Basic Energy Sciences, Materials Sciences Division, of the U.S. Department of Energy, under Contract No. DE-AC03-76SF00098. 


\section{DISCLAIMER}

This report was prepared as an account of work sponsored by an agency of the United States Government. Neither the United States Government nor any agency thereof, nor any of their employees, make any warranty, express or implied, or assumes any legal liability or responsibility for the accuracy, completeness, or usefulness of any information, apparatus, product, or process disclosed, or represents that its use would not infringe privately owned rights. Reference herein to any specific commercial product, process, or service by trade name, trademark, manufacturer, or otherwise does not necessarily constitute or imply its endorsement, recommendation, or favoring by the United States Government or any agency thereof. The views and opinions of authors expressed herein do not necessarily state or reflect those of the United States Government or any agency thereof. 


\section{DISCLAIMER}

Portions of this document may be illegible in electronic image products. Images are produced from the best available original document. 


\title{
AUTOMATED BEAM BASED ALIGNMENT OF THE ALS QUADRUPOLES*
}

\author{
G. Portmann, D. Robin, and L. Schachinger \\ Lawrence Berkeley Laboratory, Berkeley CA 94720 USA
}

\begin{abstract}
Knowing the electrical offset of the storage ring beam position monitors (BPM) to an adjacent quadrupole magnetic center is important in order to correct the orbit in the ring. We describe a simple, fast and reliable technique to measure the BPM electrical centers relative to the quadrupole magnetic centers. By varying individual quadrupole magnets and observing the effects on the orbit we were able to measure the BPM offsets in half the horizontal and vertical BPMs (48) in the ALS. These offsets were measured to an accuracy of better than $50 \mu \mathrm{m}$. The technique is completely automated and takes less than 3 hours for the whole ring.
\end{abstract}

\section{Introduction}

In a storage ring it is beneficial to have the beam orbit pass as close as possible to the center of the quadrupole magnets for a variety of reasons. First, if the beam orbit passes through the quadrupole's magnetic center, there is no steering of the orbit from the quadrupole which means that the quadrupole does not generate any orbit distortion or spurious dispersion. Second, if there is some quadrupole power supply jitter, then the amount of the orbit motion caused by the power supply jitter will be minimized. Third, the quadrupole, which is solidly anchored to a massive girder, is less sensitive to thermal effects then is the BPM which is embedded in the relatively flexible aluminum vacuum chamber.

Typically, beam position monitors (BPM) are located near the quadrupole magnets. In order to know precisely where the magnetic center of the quadrupole is, it is necessary to have a direct measurement of the relative offset between the quadrupole's magnetic center and the BPM's electrical center. Knowing of these BPM-to-quadrupole offsets allows one to correct the beam orbit to the magnetic center of the quadrupoles.

At the ALS a beam-based alignment technique has been successfully used to measure the BPM-to-quadrupole offsets of 48 of the 96 BPMs. This process, which takes about 3 hours, measures the offsets to an accuracy of better than $50 \mu \mathrm{m}$. The technique has significantly increased the absolute accuracy of the ALS BPMs since some of the measured offsets have been in excess of $1 \mathrm{~mm}$. Analysis of these measurements has also allowed us to identify magnet motion which occured between measurements.

-Work supported by the Director, Office of Energy Research, Office of Basic Energy Sciences, Materials Sciences Division, of the U.S. Department of Energy under Contract No. DEAC03-76SF00098

\section{Theory}

By changing the strength of an individual quadrupole one can vary the closed orbit of the beam. The change in the closed orbit depends upon how much the beam's closed orbit is offset from the quadrupoles magnetic center. For the case of a perfectly linear machine without coupling, the effect on the transverse orbit at a location $s$ from a change in the strength of a thin lens quadrupole located at longitudinal position $\bar{s}$ is

$$
\begin{aligned}
& \Delta \mathrm{x}(s)=\left(\frac{\Delta k(\bar{s}) x(\bar{s})}{B \rho}\right)\left(\frac{1}{1-k(\bar{s}) \frac{k \beta(\sigma)}{2 B \rho t \Delta \mathrm{A} \pi \nu}}\right) \\
& \times \quad \frac{\sqrt{\beta(\mathrm{s})} \sqrt{\beta(\bar{s})}}{2 \sin \pi \nu} \cos (\varphi(\mathrm{s})-\varphi(\bar{s})-\pi \nu) .
\end{aligned}
$$

where $\beta(\bar{s})$ and $\beta(s)$ are the beta function at the location of the quadrupole and observation point respectively, $\Delta k(\bar{s})$ is the change in the integrated strength of the quadrupole, $x(\bar{s})$ is the position of the beam orbit in the quadrupole, $B \rho$ is the magnetic rigidity of the beam, $\nu$ is the tune of the ring, and $\varphi(\bar{s})$ and $\varphi(s)$ are the betatron phases at the location of the quadrupole and observation point respectively.

From equation 1 one can see that the change in orbit $\Delta \mathrm{x}(s)$ from a change in quadrupole strength is a linear function of the offset of the orbit in the quadrupole $x(\bar{s})$. By changing the strength of the quadrupole and observing the change in the orbit at the BPMs one can in principle determine the offset of the orbit in that quadrupole. However in order to compute the offset of the orbit directly from this equation, one needs to know the beta functions, tune and phase advance, as well as the change in quadrupole strength very precisely. Fortunately, the magnetic center of the quadrupole can be located without knowing this information.

It is clear from equation 1 that if the orbit goes though the center of the quadrupole then a subsequent change in the strength of that quadrupole will have no effect on the orbit. Therefore, the center of the that quadrupole can be located by adjusting the beam orbit in that quadrupole to such a position where a variation of that quadrupole's strength will create a minimal distortion of the beam orbit. After placing the beam in the center of the quadrupole, the reading of the adjacent BPM is the relative quadrupoleto-BPM offset. This method was used to determine the BPM-to-quadrupole offsets in the ALS.

In order to use this method one must be able to individually adjust the strength of the quadrupole magnet. It is also important that the BPM is located near in betatron 
phase to the quadrupole, because even if the beam is passing through the center of the quadrupole it may be passing through at an angle. This angle can produce a large error in our measurement of the offset if the betatron phase is large. Therefore it is only valid to look at BPMs that are located at positions close in phase to the quadrupole. The following section will explain how this technique is implemented in the ALS storage ring.

\section{Experimental Technique}

The ALS has 3 families of quadrupoles: QF, QD, and QFA. All the quadrupoles in the QF and QD families have individually adjustable power supplies. Unfortunately, the quadrupoles in the QFA family are powered in series off one common supply, hence the algorithm described in the previous section will not work for this particular family. It may be possible to adapt the algorithm for magnets powered in series, however this has not yet been attempted at the ALS. Other facilities have used backleg windings, [1], or shunts resistors, [2], to overcome this problem.

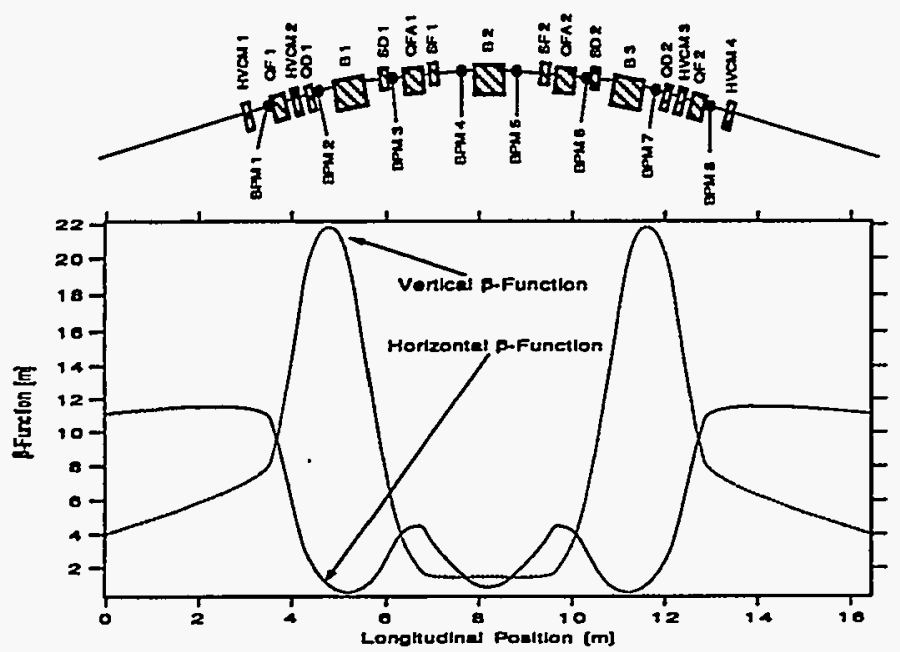

Figure. 1. Typical ALS Sector: Layout (Top) and Beta Functions (Bottom)

Each of the 12 sectors in the ALS contains 2 QF, 2 QD and 2 QFA quadrupoles. The layout of a typical sector is shown in Fig. 1. Next to each QF and QD quadrupole is a BPM. (BPM 1 is next to QF1, BPM 2 is next to QD1, BPM 7 is next to QD2, and BPM 8 is next to QF2.) Therefore in each sector one can find the relative quadrupole to BPM offset in four quadrupole-BPM pairs. A typical straight section contains four horizontal correctors (HCM) and four vertical correctors (VCM) which can be used to change the position of the orbit in the quadrupole.

The process of finding the quadrupole center is conceptually very simple. By using any corrector magnet to position the electron beam at different positions in quadrupole magnet, one determines the position which minimizes the orbit distortion when the quadrupole field is varied. This process can be automated using a simple optimization routine. There are basically two steps involved in finded the quadrupole magnet center: first change the orbit in a par- ticular quadrupole, then measure the orbit distortion from varying the quadrupole strength. These steps are repeated several times in order to find the position of the beam orbit which creates the minimum orbit distortion. To measure the orbit distortion due to a quadrupole's strength variation, the difference in closed orbit is measured at two quadrupole setpoints - one below the nominal setpoint and the other above. The merit function for the search is

$$
f\left(\theta_{c m}\right)=\frac{1}{96} \sum_{i=1}^{96}\left(x_{i}(+\Delta k)-x_{i}(-\Delta k)\right)^{2},
$$

where $\theta_{c m}$ is the strength of the corrector magnet, $x_{i}(\Delta k)$ is the orbit after changing the quadrupole by $\Delta k$, and $i$ is the BPM number. The sum goes from 1 to 96 because the ALS has 96 BPMs.

For example, in order to measure the orbit distortion from a quadrupole in the horizontal plane, one would do the following. First, change the strength of the quadrupole by $\Delta k$ so that the strength is now $k+\Delta k$ and measure the horizontal orbit in all of the BPMs $\left(x_{i}(+\Delta k)\right)$. Then, change the strength to $k-\Delta k$ and again measure the horizontal orbit $\left(x_{i}(-\Delta k)\right)$. Finally, compute equation 2 .

The ideal size of $\Delta k$ will be machine-dependent. If $\Delta k$ is large, the merit function will be "better," i.e. higher signal-to-noise ratio from the BPMs, but the quadrupole hysteresis effects will be large. The hysteresis does not affect the merit function accuracy very much, but it does adversely affect the machine tune since the merit function is computed many times. The change in quadrupole currents for this experiment were chosen based on trial-anderror \pm 3 amps for $Q F$ and \pm 5 amps for $Q D$. Due to the hysteresis effect, the quadrupoles need to be reconditioned after approximately 16 magnetic centers are found.

In order to find the quadrupole magnet center, one has to find the optimal corrector setting which minimizes equation 2. The functional form of this equation, ignoring highorder effect, is quite easy to find. The position of the orbit in the quadrupole is a linear function of corrector strength (i.e. $x_{Q U A D} \propto \theta_{\mathrm{cm}}$ ). From equation 1 , the change in the orbit from a quadrupole is proportional to the orbit at the quadrupole (i.e. $x_{i}(\Delta k) \propto x_{Q U A D}$ ). Therefore, the orbit due to a change in the quadrupole field is directly proportional to the current in the corrector.

$$
x_{i}(\Delta k) \propto \theta_{c m} .
$$

Because the merit function, $f\left(\theta_{c m}\right)$, is a quadratic function of the orbit in the BPM (see equation 2), $f\left(\theta_{c m}\right)$ will be a quadratic function of corrector strength, $\theta_{c m}$, and thus will be shaped like a parabola

$$
f\left(\theta_{c m}\right) \propto \theta_{c m}^{2} .
$$

There are a number of ways to find the minimum of this function. Theoretically, one could empirically measure three points and compute the parabola. The problem is that BPM and corrector magnet noise corrupts the merit function calculation. However, by choosing corrector magnet setpoints that are relatively far apart and measuring 
the merit function at "many" locations, one can obtain a very accurate quadrupole center measurement (see figure 2 ). At the ALS, by changing the corrector current in discrete steps, approximately .75 amps per step, and performing a least squares fit of seven data points to a parabola, the center of the quadrupole was calculated to an accuracy of at least \pm 50 microns.

A gradient search method of finding the minimum of the merit function was briefly tried. However, it was difficult to "fine tune" a gradient method because of BPM and corrector magnet noise. Basically, when performing a gradient search algorithm it is possible for two measurement points (corrector currents) to be very close together, which can produce an erroneous gradient calculation due to measurement noise corrupting the merit function. Since the discrete step, least squares fit methods works so well, the gradient search method was abandoned.

For each quadrupole the vertical and horizontal offsets were measured separately-the vertical was measured first and then the horizontal. Each quadrupole magnet was paired with the most effective corrector in each plane by examining the phase advance and beta functions in an attempt to maximize the change in orbit at the quadrupole. Due to lattice geometry the effectiveness of the corrector varies with quadrupole family.

\section{A. Example: Sector 3, QF1 and BPM1}

Figure 2 shows the experimental results for quadrupole QF1 in sector 3. Here the BPM-to-quadrupole offset determined was between QF1 and BPM1. VCM1 and HCM1 in sector 3 were used to change the orbit vertically and horizontally in QF1 and BPM1. Figure 2 shows the merit function vs. corrector current. The algorithm calculates seven points along the merit function, $f\left(\theta_{\mathrm{cm}}\right)$, guaranteeing that there are three points on either side of the minimum point. The raw data points are depicted as circles in the figure. The solid line is the seven point curve fit to a parabola and the " $\mathrm{X}$ " is the minimum of the function. A final data point is taken at the minimun to verify that the minimum has been reached. The data points lie nicely on a parabola which is expected from equation 4 .

Figure 2 shows the BPM reading vs. the same corrector currents. As expected, the position at the BPM is a linear function of corrector current. The offset between BPM1 and QF1 is located at the " $\mathrm{X}$ " on the dashed line in Figure 2. The BPM data was also fit using the least-squares method to a line to further reduce BPM noise. Thus when the beam was passing through the center of the quadrupole, the BPM read $0.9 \mathrm{~mm}$ horizontally and $0.4 \mathrm{~mm}$ vertically.

\section{Conclusion}

This method of finding the BPM-to-quadrupole positional offsets has proven to be reliable and relatively fast. The accuracy of the measurement is sensitive to BPM and power supply noise, however, the effects from these error sources can be substantially mitigated by increasing the number of points measured. The number of points and the range of power supply currents used in the corrector and
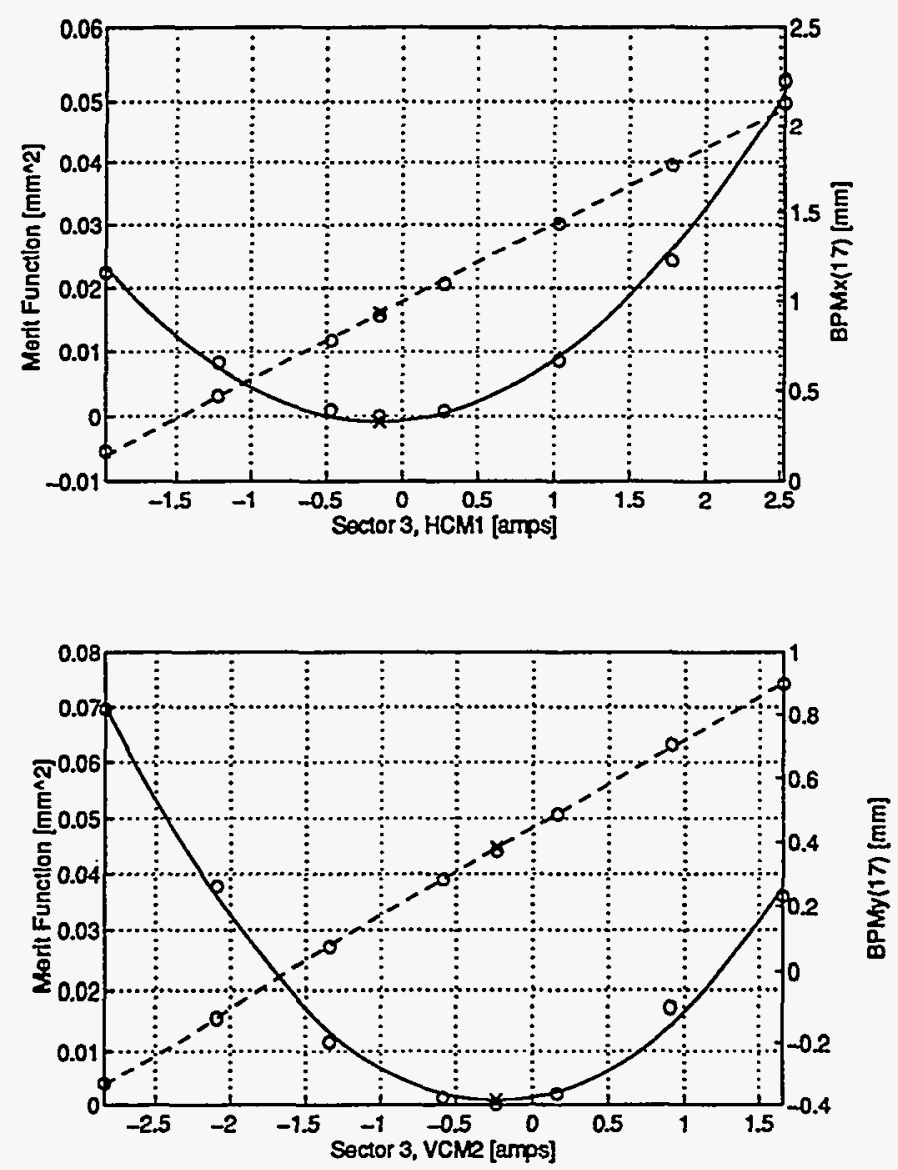

Figure. 2. Merit function (solid) and BPM (dashed) values verses corrector strength for QF1 in Sector 3

quadrupole magnets are machine dependent, but it is quite easy to adjust these parameters. Since the BPM offsets in the ALS storage ring are typically between $.25-1.25 \mathrm{~mm}$, [4], this technique has yielded essential data for correcting the closed orbit in the ALS.

\section{Acknowledgements}

The authors would like to thank A. Jackson for his support and encouragement during this study.

\section{References}

[1] I. Barnett et al., "Dynamic Beam Based Alignment"CERN-SL 9484 (BI).

[2] P. Rojsel, "A Beam Position Measurement System Using Quadrupole Magnets Magnetic Centra as the Position Reference," Nuc. Inst. Meth. in Phys. Res., 343, (1994).

[3] M. Sands, "The Physics of Electron Storage Rings: an Introduction", Stanford Linear Accelerator Note, SLAC-121, (1970), 53.

[4] G. Portmann and D. Robin, "Beam Based Alignment of the Quadrupole Centers in the ALS," LSAP Note194 (1995). 